\title{
Cedecea lapagei in a Patient with Multiple Injuries: Report of a Rare Case
}

\author{
Xiaofan $\mathrm{Xu}^{1 *}$, Kaiyue Chang ${ }^{*}$, Dongxue Song1* ${ }^{*}$, Guangtong Tian ${ }^{2 *}$, \\ Xiaohang $\mathrm{Hu}^{3}$, Liqing Jiang 3 , Bin Zhang3, Qingli Bie ${ }^{3}$, Shuhua $\mathrm{Lu}^{3}$, \\ Xiaozhe $\mathbf{L i}^{3}$, Haixin Dong ${ }^{3}$, Chengqiang Jin ${ }^{3 \#}$
}

${ }^{1}$ School of Clinical Medicine, Jining Medical University, Jining, China

${ }^{2}$ Department of Clinical Laboratory, Jining Hospital of traditional Chinese Medicine, Jining, China ${ }^{3}$ Department of Clinical Laboratory, The Affiliated Hospital of Jining Medical University, Jining, China

Email: "jincq2008@163.com

How to cite this paper: Xu, X.F., Chang, K.Y., Song, D.X., Tian, G.T., Hu, X.H., Jiang, L.Q., Zhang, B., Bie, Q.L., Lu, S.H., Li, X.Z., Dong, H.X. and Jin, C.Q. (2021) Cedecea lapagei in a Patient with Multiple Injuries: Report of a Rare Case. Journal of Biosciences and Medicines, 9, 1-5. https://doi.org/10.4236/jbm.2021.911001

Received: September 28, 2021

Accepted: October 26, 2021

Published: October 29, 2021

Copyright $\odot 2021$ by author(s) and Scientific Research Publishing Inc. This work is licensed under the Creative Commons Attribution International License (CC BY 4.0).

http://creativecommons.org/licenses/by/4.0/

\begin{abstract}
Cedecea lapagei (C. lapagei) is an opportunistic pathogen in old patients with many comorbid diseases and the immunosuppressed. It is a gram-negative, facultative anaerobe bacterium of the Enterobacteriaceae family. We present a rare case of a patient with multiple injuries, $C$. lapagei was found from the exudate of the wound, what's more, the Proteus vulgaris was also found in blood culture medium at the same time. According to the available literature, this is the first case of simultaneous infection of two bacteria including C. lapagei and Proteus vulgaris from the exudate of the traumatic wound.
\end{abstract}

\section{Keywords \\ C. lapagei, Cedecea, Proteus vulgaris, Exudate}

\section{Introduction}

The genus Cedecea belongs to the family Enterobacteriaceae. Cedecea consists of six species and three of these species are known human pathogens: Cedecea davisae, Cedecea lapagei, and Cedecea neteri [1]. Although discovered in 1977, it was not until the year 2006 that the species C. lapagei became known as a pathogenic bacterium [2]. It has been isolated in sputum, BAL specimen, blood, etc. [3] [4], but it has not been found in exudate until now. A strain of C. lapagei was detected in the exudate from the wounded inpatient by clinic laboratory of our hospital. More important, it's a mixed infection with Proteus vulgaris.

*These authors contributed equally to this work.

${ }^{\#}$ Corresponding author. 


\section{Case Report}

The male patient, 42 years old, a worker, was sent to our hospital due to a motorcycle fall 6 hours ago. In the emergency department, he was admitted to our hospital's hand and foot surgical ward with "multiple injuries, multiple open fractures and dislocations of the right foot, multiple rupture of blood vessels, nerves and tendons of the right foot, skin and soft tissue defects of the right foot, pelvic fracture, hematoma of the right ilium, and fracture of the right fibula head". The patient has clear mind, poor spirit, no obvious abnormality in heart and lung, and no water intake. He used to be in good health and had a personal history of 20 cigarettes/day for 20 years, 200 gram/day alcohol consumption for 10 years.

There was no family genetic disease or infectious history. On the day of admission, the patient was given surgical treatment. After the operation, the patient was treated with anti-bacterial, detumescence, fracture promotion, wound healing, anticoagulation, antispasmodic, and heat preservation with baking lamp. Although the wounds were well matched, there was some more liquid exudation. The patient was given iodophor disinfection, aseptic auxiliary material replacement, and the exudate was taken for bacterial culture and drug sensitivity test. Informed consent was obtained from the patient.

\section{Bacterial Culture and Identification}

The exudate was inoculated into blood culture medium and Chinese blue culture medium, and cultured in $36^{\circ} \mathrm{C}$ incubator for 24 hours. There were small round colonies with gray white, moist and smooth edges, without hemolytic ring. The microscopic examination showed Gram-negative bacteria. In addition, there were migratory membrane colonies in the blood culture medium. The round colonies and membranous colonies were identified as C. lapagei and Proteus vulgaris by Vitek ${ }^{\text {tw }} 2$ compact system (BioMérieux, France) following the manufacturer's instructions.

\section{Drug Sensitivity Test}

Antimicrobial susceptibility testing was determined by the automated Vitek ${ }^{\text {tix }} 2$ compact system using AST-GN13 susceptibility cards. One hundred and forty-five microliter bacterial suspension of a $0.5-\mathrm{McF}$ arl and turbidity was mixed with $3 \mathrm{~mL} 0.45 \% \mathrm{NaCl}$ solution. The AST-GN13 card filled with the mixture was used. The E. coli ATCC25922 was used as quality control. Drug sensitivity test showed that $C$. lapagei was resistant to Ampicillin, cefazolin, imipenem and Sulfamethoxazo. It was mediated to ampicillin/sulbactam, gentamycin, tobramycin, ciprofloxacin and levofloxacin (see Table 1). The drug sensitivity test of Proteus vulgaris showed that it was resistant to ampicillin, cefazolin, imipenem, amikacin, gentamicin, ciprofloxacin, sulfamethoxazo and sensitive to piperacillin/tazobactam, cefotaxime, ceftazidime, cefatriaxone, cefepime, aztreonam, ertap nan, levofloxacin (see Table 2). 
Table 1. Drug sensitivity test of $C$. lapagei.

\begin{tabular}{ccc}
\hline No. & Antibiotics & $\mathrm{S} / \mathrm{R} / \mathrm{I}(\mathrm{MIC}, \mu \mathrm{g} / \mathrm{ml})$ \\
\hline 1 & Ampicillin & $\mathrm{R}(\geq 32)$ \\
2 & Ampicillin/sulbactam & $\mathrm{I}(16)$ \\
3 & Piperacillin/tazobactam & $\mathrm{S}(\leq 4)$ \\
4 & Cefazolin & $\mathrm{R}(\geq 64)$ \\
5 & Cefotaxime & $\mathrm{S}(\leq 4)$ \\
6 & Ceftazidime & $\mathrm{S}(4)$ \\
7 & Cefatriaxone & $\mathrm{S}(\leq 1)$ \\
8 & Cefepime & $\mathrm{S}(\leq 1)$ \\
9 & Aztreonam & $\mathrm{S}(\leq 1)$ \\
10 & Ertap Nan & $\mathrm{S}(\leq 0)$ \\
11 & Imipenem & $\mathrm{R}(4)$ \\
12 & Amikacin & $\mathrm{I}(\leq 2)$ \\
13 & Gentamicin & $\mathrm{I}(8)$ \\
14 & Tobramycin & $\mathrm{I}(8)$ \\
15 & Ciprofloxacin & $\mathrm{I}(2)$ \\
16 & Levofloxacin & $\mathrm{I}(4)$ \\
17 & Sulfamethoxazo & $\mathrm{R}(\geq 32)$ \\
\hline & & $\mathrm{S}$ \\
\hline
\end{tabular}

Note: MIC, minimum inhibitory concentration $(\mu \mathrm{g} / \mathrm{ml})$; R, resistant; $\mathrm{S}$, susceptible; I, intermediate.

Table 2. Drug sensitivity test of Proteus vulgaris.

\begin{tabular}{ccc}
\hline No. & Antibiotics & $\mathrm{S} / \mathrm{R} / \mathrm{I}(\mathrm{MIC}, \mu \mathrm{g} / \mathrm{ml})$ \\
\hline 1 & Ampicillin & $\mathrm{R}(\geq 32)$ \\
2 & Ampicillin/sulbactam & $\mathrm{R}(\geq 32)$ \\
3 & Piperacillin/tazobactam & $\mathrm{S}(\leq 4)$ \\
4 & Cefazolin & $\mathrm{R}(\geq 64)$ \\
5 & Cefotaxime & $\mathrm{S}(\leq 4)$ \\
6 & Ceftazidime & $\mathrm{S}(\leq 1)$ \\
7 & Cefatriaxone & $\mathrm{S}(\leq 1)$ \\
8 & Cefepime & $\mathrm{S}(\leq 1)$ \\
9 & Aztreonam & $\mathrm{S}(\leq 1)$ \\
10 & Ertap Nan & $\mathrm{S}(\leq 0)$ \\
11 & Imipenem & $\mathrm{R}(4)$ \\
12 & Amikacin & $\mathrm{R}(\geq 64)$ \\
13 & Gentamicin & $\mathrm{R}(\geq 16)$ \\
14 & Tobramycin & $\mathrm{I}(8)$ \\
15 & Ciprofloxacin & $\mathrm{R}(\geq 4)$ \\
16 & Levofloxacin & $\mathrm{S}(2)$ \\
17 & Sulfamethoxazo & $\mathrm{R}(\geq 32)$ \\
\hline
\end{tabular}

Note: MIC, minimum inhibitory concentration $(\mu \mathrm{g} / \mathrm{ml})$; $\mathrm{R}$, resistant; $\mathrm{S}$, susceptible; I, intermediate. 


\section{Prognosis and Outcome}

After changing fresh dressing for the wound once a day and constant anti-infection treatment with the addition of levofloxacin, the exudate of the wound was continuously reduced apparently. After three days, the subsequent bacterial culture and identification showed that there was no bacterial growth in the exudate. The patient was well recovered and discharged soon afterwards.

\section{Discussion}

C. lapagei is a member of the Enterobacteriaceae family and is an uncommon opportunistic pathogen. Its susceptible population is individuals with low or suppressed immune function, such as patients with granulocytopenia, tumor chemotherapy, organ transplantation, large-scale surgery or trauma [2] [5], such as it can follow cement-related chemical burn injury [6]. There are very few reports of isolation of this organism from human biological samples; it is also found to be a pathogen in elderly or otherwise life-threatening conditions [7], for example in a patient with chronic obstructive pulmonary disease [8]. Biswal et al. presented a rare case of a patient with underlying malignancy of buccal mucosa, who developed an oral ulcer superinfected with $C$. lapagei and this is the first case of $C$. lapagei from India detected in a cancer patient [9]. C. lapagei can also cause ventilator-associated pneumonia and sepsis in Neonatal Intensive Care Unit [10].

We presented the first rare case of simultaneous infection of two bacteria including C. lapagei and Proteus vulgaris from the exudate of the traumatic wound in the world. But their relevance between C. lapagei and Proteus vulgaris has yet to be elucidated. It was reported that $C$. lapagei can produce New Delhi metallo- $\beta$-lactamase-1 (NDM-1) in some certain areas [11]. NDM-1 is a carbapenemase able to hydrolyze nearly all available $\beta$-lactam antibiotics, endangering efficacious antibacterial treatments [12]. Further studies are needed to understand its biology characteristics and the role in the mode of transmission, the spectrum of infection, and treatment options. There is a great need for physicians to cognize the emerging pathogens and know their antibiotic resistance profiles.

\section{Acknowledgements}

This work was supported by the project of science and technology development research center of ministry of health (Grant number: 28-10-2), the Natural Science Foundation of Shandong Province (Grant number: ZR2010HL038), Jining Municipal Science and Technology development projects (Grant number: 2012jnjc16 \& 2014jnnk23), Shandong provincial medical and health science and technology development project (Grant number: 2013WS033), Shandong Province Traditional Chinese medicine science and technology development project (Grant number: 2015-244), and the Project of Shandong Province Higher Educational Science and Technology Program (Grant number: J15LL11). Nursery Program of Affiliated Hospital of Jining Medical University (Grant number: MP-2018-008). 


\section{Conflicts of Interest}

The authors declare no conflicts of interest regarding the publication of this paper.

\section{References}

[1] Chavez, V.R., Rosas De Silva, M.F., Alcaraz, H.O., et al. (2018) Death Related to $C$. lapagei in a Soft Tissue Bullae Infection: A Case Report. Journal of Medical Case Reports, 12, 328. https://doi.org/10.1186/s13256-018-1866-X

[2] Davis, O. and Wall, B.M. (2006) "Broom Straw Peritonitis" Secondary to C. lapagei in a Liver Transplant Recipient. Peritoneal Dialysis International, 26, 512-513. https://doi.org/10.1177/089686080602600422

[3] Yetkin, G., Ay, S., Kayabas, U., Gedik, E., Gucluer, N. and Caliskan, A. (2008) A Pneumonia Case Caused by C. lapagei. Mikrobiyoloji Bülteni, 42, 681-684.

[4] Ramaswamy, V.V., Gummadapu, S. and Suryanarayana, N. (2019) Nosocomial Pneumonia and Sepsis Caused by a Rare Organism C. lapagei in an Infant and a Review of Literature. BMJ Case Reports, 12, e229854. https://doi.org/10.1136/bcr-2019-229854

[5] Lopez, L.A., Ibarra, B.S., de la Garza, J.A., Rada Fde, J., Nunez, A.I. and Lopez, M.G. (2013) First Reported Case of Pneumonia Caused by C. lapagei in America. Brazilian Journal of Infectious Diseases, 17, 626-628. https://doi.org/10.1016/j.bjid.2013.03.003

[6] Dalamaga, M., Karmaniolas, K., Arsenis, G., Pantelaki, M., Daskalopoulou, K., Papadavid, E. and Migdalis, I. (2008) C. lapagei Bacteremia Following Cement-Related Chemical Burn Injury. Burns, 34, 1205-1207. https://doi.org/10.1016/j.burns.2007.09.001

[7] Kanakadandi, V.S., Sarao, M.S. and Cunningham, J.M. (2019) A Rare Case of Cedecea Davisae Bacteremia Presenting as Biliary Sepsis. Cureus, 11, e5298. https://doi.org/10.7759/cureus.5298

[8] Hong, S.K., Lee, J.S. and Kim, E.C. (2015) First Korean Case of C. lapagei Pneumonia in a Patient with Chronic Obstructive Pulmonary Disease. Annals of Laboratory Medicine, 35, 266-268. https://doi.org/10.3343/alm.2015.35.2.266

[9] Biswal, I., Hussain, N.A. and Grover, R.K. (2015) C. lapagei in a Patient with Malignancy: Report of a Rare Case. Journal of Cancer Research and Therapeutics, 11, 646. https://doi.org/10.4103/0973-1482.147736

[10] Kury, C.M.H., Yabrudi, A.A., de Souza, T.B., et al. (2017) First Reported Case of Ventilator-Associated Pneumonia and Sepsis Caused by C. lapagei in a Brazilian Neonatal Intensive Care Unit. Journal of the Pediatric Infectious Diseases Society, 6 , 209-210. https://doi.org/10.1093/jpids/piw077

[11] Ahmad, N., Ali, S.M. and Khan, A.U. (2017) First Reported New Delhi Metallo-Beta-Lactamase-1-Producing C. lapagei. International Journal of Antimicrobial Agents, 49, 118-119. https://doi.org/10.1016/j.ijantimicag.2016.10.001

[12] Linciano, P., Cendron, L., Gianquinto, E., Spyrakis, F. and Tondi, D. (2019) Ten Years with New Delhi Metallo-Beta-Lactamase-1 (NDM-1): From Structural Insights to Inhibitor Design. ACS Infectious Diseases, 5, 9-34.

https://doi.org/10.1021/acsinfecdis.8b00247 